\title{
Quantification of Inter-Limb Symmetries With Rate of Force Development and Relaxation Scaling Factor
}

\author{
Darjan Smajla ${ }^{1,2}$, Jure Žitnik ${ }^{1,2}$ and Nejc Šarabon ${ }^{1,2,3,4 *}$
}

${ }^{1}$ Faculty of Health Sciences, University of Primorska, Izola, Slovenia, ${ }^{2}$ Human Health Department, InnoRenew CoE, Izola, Slovenia, ${ }^{3}$ Andrej Marušič Institute, University of Primorska, Koper, Slovenia, ${ }^{4}$ S2P, Science to Practice, Ltd., Laboratory for Motor Control and Motor Behavior, Ljubljana, Slovenia

The inter-limb (a)symmetries have been most often assessed with the tests that quantify the maximal muscle capacity. However, the rapid force production and relaxation during submaximal tasks is equally important for successful sports performance. This can be evaluated with an established rate of force development and relaxation scaling factor (RFD-SF/RFR-SF). The aims of our study were (1) to assess the intrasession reliability of shortened RFD-SF/RFR-SF protocol and its absolute and symmetry outcome measures, (2) to compare the main absolute RFD-SF/RFR-SF outcome measures (slopes of RFD-SF and RFR-SF: $k_{R T D}-S F$ and $k_{R F R-S F}$, theoretical peak

OPEN ACCESS

Edited by:

G. Gregory Haff,

Edith Cowan University, Australia

Reviewed by:

Stuart Nathan Guppy Edith Cowan University, Australia Gennaro Boccia,

University of Turin, Italy

*Correspondence: Nejc Šarabon nejc.sarabon@fvz.upr.si

Specialty section: This article was submitted to Exercise Physiology, a section of the journal Frontiers in Physiology

Received: 11 March 2021 Accepted: 21 May 2021

Published: 21 June 2021

Citation:

Smajla D, Žitnik J and Šarabon N (2021) Quantification of Inter-Limb Symmetries With Rate of Force Development and Relaxation Scaling Factor. Front. Physiol. 12:679322. doi: 10.3389/fphys.2021.679322
RFD/RFR: TP $\mathrm{RFD}_{\text {and }}$ TPRFR across gender and sports groups, and (3) to compare inter-limb symmetries across gender and sports groups for main outcome measures ( $\mathrm{k}_{\mathrm{RFD}-\mathrm{SF}}, \mathrm{k}_{\mathrm{RFR}-\mathrm{SF}}, \mathrm{TP}_{\mathrm{RFD}}$, and TPRFR$)$. A cross-sectional study was conducted on a group of young health participants (basketball and tennis players, and students): 30 in the reliability study and 248 in the comparison study. Our results showed good to excellent relative and excellent absolute reliability for the selected absolute and symmetry outcome measures ( $k_{R F D}-S F, k_{R F R-S F}, P_{R F D}$, and TPRFR). We found significantly higher absolute values for $\mathrm{k}_{\mathrm{RFD}-\mathrm{SF}}$ and $\mathrm{TP}_{\mathrm{RFD}}$ in males compared to females for the preferred ( $k_{R F D}-S F: 9.1 \pm 0.9$ vs. $\left.8.6 \pm 0.9 / s\right)$ and the non-preferred leg (k $k_{R F D}-S F$ : $9.1 \pm 0.9$ vs. $8.5 \pm 0.8 / \mathrm{s}$ ), while there was no effect of sport. Significantly lower symmetry values for $\mathrm{k}_{\mathrm{RFR}-\mathrm{SF}}(88.4 \pm 8.6$ vs. $90.4 \pm 8.0 \%)$ and TPRFR $(90.9 \pm 6.8$ vs. $92.5 \pm 6.0 \%$ ) were found in males compared to females. Moreover, tennis players had significantly higher symmetry values for $\mathrm{K}_{\mathrm{RFR}-\mathrm{SF}}(91.1 \pm 7.7 \%)$ and $\operatorname{TP}_{\mathrm{RFR}}(93.1 \pm 6.0 \%)$ compared to basketball players (kRFR-SF: $88.4 \pm 8.7 \%$ and TPRFR: $90.9 \pm 6.7 \%$ ) and students (KRFR-SF: $87.6 \pm 8.7 \%$ and TPRFR: $90.5 \pm 6.7 \%$ ). Our results suggest that the reduced RFD-SF/RFR-SF protocol is a valuable and useful tool for interlimb (a)symmetry evaluation. Differences in symmetry values in $k_{R F R-S F}$ and TPRFR (relaxation phase) were found between different sports groups. These may be explained by different mechanisms underlying the muscle contraction and relaxation. We suggest that muscle contraction and relaxation should be assessed for in-depth inter-limb symmetry investigation.

Keywords: rapid movement, asymmetry, force, relaxation, basketball, tennis, rapid strength 


\section{INTRODUCTION}

Comparing the ability of one limb in contrast to another is a popular research topic from various aspects. Inter-limb asymmetries have been most commonly studied in athletes in relation to sports performance (Sato and Heise, 2012; Lockie et al., 2014; Dos'Santos et al., 2017; Bishop et al., 2018b; Madruga-Parera et al., 2019) and injury risk (Croisier et al., 2008; Lehance et al., 2009; Green et al., 2018). The inter-limb asymmetries have been most often assessed using tests requiring maximal performance such as strength (Fousekis et al., 2010), jumping (Bishop et al., 2020), and change of direction speed tests (Dos'Santos et al., 2017).

To quantify the maximal capacity of the quadriceps muscle, commonly isometric and isokinetic testing protocols are used (Wilson and Murphy, 1996; Croisier et al., 2007; Maffiuletti et al., 2007). Maffiuletti et al. (2010) suggested that rapid force production during voluntary tasks is better related to specific sports performance than maximal force. Furthermore, it is well known that rapid force production is typically required in most sports-specific movements. Capacity of the neuro-muscular system for rapid strength production has been conventionally quantified by rate of force development (RFD) during maximal force production levels. The RFD during maximal voluntary contraction (MVC) is conventionally used to evaluate rapid strength (Knezevic et al., 2014; Mirkov et al., 2017), but, in various sports situations there is a demand for quick submaximal force production, especially in tasks that require precision (Thorlund et al., 2009).

To evaluate inter-limb differences for rapid force production during different submaximal exercises, the rate of force development scaling factor (RFD-SF) was proposed as a measure with higher ecological validity than the RFD (Boccia et al., 2018). In the RFD-SF protocol, the participant needs to perform rapid isometric contractions corresponding to different submaximal percentages of the previously assessed maximal force during MVC. Previous methodological studies recommended performing at least 120 rapid isometric pulses at different force amplitudes for a valid RFD-SF assessment (Bellumori et al., 2011; Djordjevic and Uygur, 2017). The RFD generated during each rapid isometric pulse provides a measure of the rapid force production ability at each of different submaximal amplitudes. The relationship between peak forces and the corresponding RFDs is represented by the slope of these relationships, specifically the slope of the regression line interpolated on the peak force: RFD scatter plot $\left(\mathrm{k}_{\mathrm{RFD}}-\mathrm{SF}\right)$. This measure enables the investigation of interlimb asymmetries at movement initiation and quickness of force production, which has been investigated only by two studies to date (Boccia et al., 2018; Smajla et al., 2020). Smajla et al. (2020) showed that leg preference has no effect on $\mathrm{k}_{\mathrm{RFD}-\mathrm{SF}}$, while Boccia et al. (2018) reported that $\mathrm{k}_{\mathrm{RFD}-\mathrm{SF}}$ has been shown as a more sensitive tool for identifying interlimb asymmetry compared to a classical isokinetic test (Boccia et al., 2018). However, both studies were conducted on a small number of participants (up to 40). Recently, one of the studies showed that to assess the ability of quick force relaxation of submaximal muscle forces, the rate of force relaxation scaling factor (RFR-SF) can also be determined as part of the RFD-SF protocol (Mathern et al., 2019). The slope of RFR-SF $\left(\mathrm{k}_{\mathrm{RFR}-\mathrm{SF}}\right)$ represents the regression line interpolated between peak force and the respective rate of force relaxation. RFR-SF has been shown to be impaired in people with knee osteoarthritis (Šarabon et al., 2020a) and multiple sclerosis (Uygur et al., 2020). It is well acknowledged that adequate muscle relaxation is very important for efficient performance of quick muscle actions (Kato et al., 2019). Moreover, there is clear evidence that strength, power, and speed abilities differ between sports due to specific demands (Šarabon et al., 2020b). However, there are no studies that have compared neuromuscular abilities at a submaximal level between sports and gender or assessed them from the perspective of interlimb asymmetries. It has been previously shown that $\mathrm{k}_{\mathrm{RFD}-\mathrm{SF}}$ and $\mathrm{k}_{\mathrm{RFR}-\mathrm{SF}}$ are independent of muscle size (Bellumori et al., 2011; Mathern et al., 2019). Consequently, gender differences are not expected to be found. The main outcome measures of the RFD-SF/RFR-SF protocol can provide additional insight into neuromuscular function as $\mathrm{k}_{\mathrm{RFD}-\mathrm{SF}}$ is mainly dependent on neuromuscular activation mechanisms (Folland et al., 2014), whereas $\mathrm{k}_{\mathrm{RFR}-\mathrm{SF}}$ is mainly influenced by intrinsic properties of the muscle (Mathern et al., 2019). For this reason, such assessment can be valuable for inter-limb asymmetries exploration. Despite a large number of studies that have assessed the magnitude of inter-limb asymmetries using different measures (Sato and Heise, 2012; Maloney et al., 2016; Bishop et al., 2019c; Dos'Santos et al., 2019), there is limited evidence on the variation in inter-limb asymmetries across athletes in different sports.

In our previous work, we found the reduced RFD-SF/RFR-SF protocol (36 pulses) to be reliable for quantifying neuromuscular abilities to quickly produce and relax muscle force during submaximal contraction compared to the standard protocol (100-125 pulses), while introducing a new outcome measure in the form of theoretical maximal RFD/RFR ( $\mathrm{TP}_{\mathrm{RFD}}$ and $\mathrm{TP}_{\mathrm{RFR}}$ ) (Smajla et al., 2021b). Therefore, the aims of our study were as follows. First is to assess the intra-session reliability of the absolute RFD-SF/RFR-SF outcome measures and the inter-limb symmetries of the reduced protocol. We hypothesized that all outcome measures would have good to excellent reliability with the exception of the $y$-intercept. Second is to compare the absolute main outcome measures of interest $\left(\mathrm{k}_{\mathrm{RFD}-\mathrm{SF}}\right.$, $\mathrm{k}_{\mathrm{RFR}-\mathrm{SF}}, \mathrm{TP}_{\mathrm{RFD}}$, and $\left.\mathrm{TP}_{\mathrm{RFR}}\right)$ across gender and sports groups (basketball players, tennis players, and students from Faculty of Sports). We hypothesized that similar results would be found in males and females, while significant differences between sports groups (basketball players, tennis players, and students of Faculty of Sports) would be found for the main outcome measures. Third is to compare inter-limb symmetries across genders and sports groups for main outcome measures of interest $\left(\mathrm{k}_{\mathrm{RFD}-\mathrm{SF}}, \mathrm{k}_{\mathrm{RFR}-\mathrm{SF}}, \mathrm{TP}_{\mathrm{RFD}}\right.$, and $\left.\mathrm{TP}_{\mathrm{RFR}}\right)$. We hypothesized that inter-limb symmetries calculated for main outcome measures would not differ between genders, while lower symmetry would be found in basketball players compared to students and tennis players. 


\section{MATERIALS AND METHODS}

\section{Participants}

A total of 248 (152 male and 96 female) participants volunteered for the cross-sectional study. Reliability analyses were conducted on a subsample group, while the differences in absolute and symmetry analyses were conducted on the entire sample (overall sample) (Table 1). Participants from basketball and tennis were included in the study if they had a training history of at least 3 years in their sports and a training frequency of at least two sessions per week in the last year. The students from Faculty of Sports were included in the study in case they had not had specific training history in the past 3 years, but they had been physically active at least two times per week in the last year (most involved in fitness and running). The sample for this study was limited to participants who reported no lower-limb injuries or low-back pain in the past 6 months. Participants with any neurological disorders were also excluded from the analysis. Leg side preference was determined by asking participants: "Which leg do you prefer when performing unilateral jumping movements?" All the participants (or their parent/guardianif participants were under 18 years of age) were informed of the testing procedures and provided an informed consent prior to study participation. Participants were instructed to avoid intense physical activity for at least $48 \mathrm{~h}$ prior to testing. The Slovenian Medical Ethics Committee (approval no. 012099/2018/5) approved the experiment, which was conducted according to the guidelines of the Declaration of Helsinki.

\section{Study Design, Tasks, and Procedures}

Each of the participants performed a knee-extension MVC with the preferred and non-preferred leg in a randomized order prior to performing the RFD-SF/RFR-SF protocol. Although torque was measured, the term force is used in this article to maintain consistency with previous literature (Bellumori et al., 2011; Mathern et al., 2019). For the MVC and RFD-SF/RFRSF protocols, participants sat in the chair of an isometric knee dynamometer (S2P, Science to Practice, Ltd., Ljubljana, Slovenia; Figure 1; Sarabon et al., 2013). The knee angle was set at $60^{\circ}$ of knee flexion (full knee extension equals $0^{\circ}$ ), while the hip angle was set at $100^{\circ}$. The knee axis was in line with the axis of the lever arm of the dynamometer. The shank support was manually adjusted for each participant so that it was positioned approximately $2 \mathrm{~cm}$ above the lateral malleolus. Hip and knee fixation was provided by rigid straps over the pelvis and the knee. For familiarization and warmup, each participant first performed two graded submaximal contractions at 50,75 , and $90 \%$ of the self-estimated maximal voluntary effort. After 3 min of rest, participants performed three maximal voluntary knee extensions with a 30 -s rest interval. They were instructed to gradually increase their torque and maintain maximal force for 3-5 s, from which peak knee extension force was determined. Participants then performed 1520 submaximal rapid contractions and relaxations performed at varying submaximal levels or until they could match the force and perform voluntary force pulses and relaxations with the required intensity. They were instructed to produce the knee extension movement as quickly as possible and then immediately relax. After familiarization, participants in the subsample group performed approximately 25-30 rapid isometric contractions at four different submaximal levels $(20,40,60$, and $80 \%$ of previously determined maximal voluntary force), for a total of 100-120 contractions. All other participants performed a reduced RFD-SF/RFR-SF protocol (Smajla et al., 2021b). We aimed for 60 pulses to be performed as some contractions might not be performed properly (slow contraction, pre-contraction of agonist or antagonist inappropriate relaxation) (Grabiner, 1994; Kamimura et al., 2009) and would be excluded from the final analysis. The target force level was displayed on a computer screen in front of the participant performing the protocol as a horizontal line on a graph. Visual feedback on the force generated by the participant during the pulse was also displayed on the screen, while participants were instructed to apply a level of force that matched the horizontal target force level displayed during each pulse. As previously suggested by Gordon and Ghez (1987), participants were instructed to focus on rapid performance rather than attempting to match force levels. There was a 60-s rest interval between two consecutive submaximal levels. Rapid pulses were elicited by an experienced examinator with a verbal command at approximately $4-5$-s intervals. The reliability and validity of the RFD-SF/RFR-SF outcome measures were first examined on a subsample of participants (Table 1). To assess the intra-session reliability in the subsample study, two sets of 36 pulses were sampled separately (nine randomly selected repetitions for each submaximal intensity) and used to calculate

TABLE 1 | Characteristics of the participants in the subsample and overall group.

\begin{tabular}{|c|c|c|c|c|c|c|c|c|c|}
\hline Sample & Group & Gender & $N$ & Age (years) & Body height (cm) & Body mass (kg) & $\begin{array}{l}\text { Left preferred } \\
\qquad(n)\end{array}$ & $\begin{array}{c}\text { Right } \\
\text { preferred }(n)\end{array}$ & $\begin{array}{c}\text { Training } \\
\text { history (years) }\end{array}$ \\
\hline Subsample & & Male & 23 & $16.5 \pm 1.0$ & $188.4 \pm 6.8$ & $77.8 \pm 10.4$ & 20 & 3 & $7.6 \pm 2.5$ \\
\hline \multirow[t]{5}{*}{ Overall } & Basketball & Male & 77 & $16.7 \pm 1.1$ & $188.0 \pm 8.0$ & $79.0 \pm 10.7$ & 65 & 12 & $7.3 \pm 2.2$ \\
\hline & & Female & 40 & $16.9 \pm 1.6$ & $175.3 \pm 5.8$ & $70.8 \pm 9.6$ & 37 & 3 & $6.8 \pm 2.5$ \\
\hline & Students & Male & 25 & $19.7 \pm 0.4$ & $182.5 \pm 5.7$ & $75.7 \pm 8.2$ & 9 & 16 & $8.3 \pm 3.8$ \\
\hline & Tennis & Male & 50 & $16.1 \pm 3.0$ & $177.1 \pm 8.4$ & $67.1 \pm 10.1$ & 36 & 14 & $8.8 \pm 3.8$ \\
\hline & & Female & 31 & $16.3 \pm 2.7$ & $169.2 \pm 5.9$ & $61.2 \pm 8.0$ & 21 & 10 & $8.1 \pm 3.7$ \\
\hline
\end{tabular}




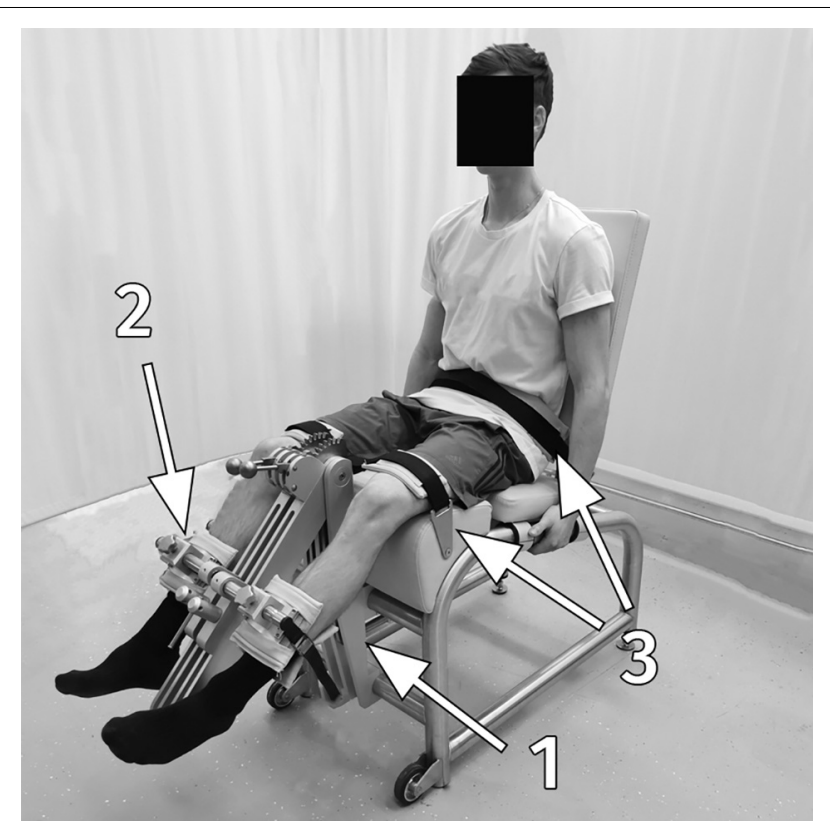

FIGURE 1 | Measurement setup; the subject in the isometric knee dynamometer: 1, a strain gage force sensor; $\mathbf{2}$, shank support; $\mathbf{3}$, rigid straps for the pelvis and knee fixation.

the outcome measures. Subsequently, all participants performed the reduced RFD-SF/RFR-SF protocol with 36 pulses to obtain the final outcome-measures data.

\section{Data Processing and Outcome Measures}

The signals from the knee dynamometer force transducer (Bending beam load cell 1-Z6FC3, Darmstadt, Germany) were sampled at $1,000 \mathrm{~Hz}$ by a custom-made LabView 2015 routine (National Instruments Corp., Austin, TX, United States). A lowpass filter (Butterworth second-order) with a $5-\mathrm{Hz}$ cut-off frequency (Djordjevic and Uygur, 2017) was used for raw data signal filtration. Another custom-made LabView 2015 routine (National Instruments Corp., Austin, TX, United States) routine was used for data analysis. The program routine automatically placed two cursors on the time derivate curve corresponding to peak RFD and peak RFR (1-ms sampling window) of each of the acquired force pulses. One additional cursor was placed on the force curve and depicted the peak force. We performed manual inspection for all signals to verify correct cursor placement. For each pulse, we recorded the magnitudes of peak force, RFD, and RFR (Mathern et al., 2019). The regression parameters were obtained from the relationship between the peak torque and corresponding peak RFD and peak RFR and used as a dependent variable. This relationship was used for the calculation of $\mathrm{k}_{\mathrm{RFD}-\mathrm{SF}}(/ \mathrm{s})$ and $\mathrm{k}_{\mathrm{RFR}-\mathrm{SF}}(/ \mathrm{s})$ of the regression line (main dependent outcome measures of interest). Other linear regression parameters, $\mathrm{r}^{2}\left(\mathrm{r}_{\mathrm{RFD}-\mathrm{SF}}^{2}, \mathrm{r}_{\mathrm{RFR}-\mathrm{SF}}^{2}\right)$, $\mathrm{y}$-intercept [y-int RFD $(\% \mathrm{MVC} / \mathrm{s}), \mathrm{y}$-int $\left._{\mathrm{RFR}}(\% \mathrm{MVC} / \mathrm{s})\right]$, and theoretical peak RFD/RFR [TP $\left.\mathrm{TPD}_{\mathrm{R}}(\% \mathrm{MVC} / \mathrm{s}), \mathrm{TP}_{\mathrm{RFR}-\mathrm{SF}}(\% \mathrm{MVC} / \mathrm{s})\right]$ were calculated for each participant. $\mathrm{TP}_{\mathrm{RFD}}$ and $\mathrm{TP} \mathrm{P}_{\mathrm{R} R}$ represent the newly introduced outcome measures determined by linear interpolation for each participant's regression line $(y=k \times x+n)$ solved for $x=100$ (maximal theoretical peak RFD/RFR). Interlimb symmetries were calculated for main outcome measures $\left(\mathrm{k}_{\mathrm{RFD}-\mathrm{SF}}, \mathrm{k}_{\mathrm{RFR}-\mathrm{SF}}, \mathrm{TP}_{\mathrm{RFD}}\right.$, and $\left.\mathrm{TP}_{\mathrm{RFR}}\right)$ in accordance with the previously published equation (Smajla et al., 2021a) (Eq. 1).

Symmetry $(\%)=100-\left(\frac{\text { higher value }- \text { lower value }}{\text { higher value }}\right) \times 100$

\section{Statistical Analysis}

R Statistical Software (version 4.0.3, R Core Team, R Foundation for Statistical Computing, Vienna, Austria) was used for all the performed statistical analyses. Descriptive statistics of the dependent variables are presented as mean \pm standard deviation. In the subsample analysis, the following reliability measures were used to describe intra-session reliability for absolute and symmetry outcome measures: (a) intraclass correlation coefficient $\left(\mathrm{ICC}_{2,1}\right)$, (b) coefficient of variation $(\mathrm{CV})$, and $\mathrm{c}$ ) typical error (TE) (Hopkins, 2000). ICC 2,1 estimates and their 95\% confidence intervals (CI) were interpreted according to Koo and Li (2015), while CV (within-subject standard deviation method) values of $<10 \%$ were considered acceptable (Cormack et al., 2008). Prior to analysis, the data distribution for each of the outcome measures in the groups under study was assessed by performing a Shapiro-Wilk test and looking at Q-Q plots of the residuals. The homogeneity of variance was determined with the use of Levene's test. A two-way analysis of variance (ANOVA) was used to examine the effect of gender and sports on main outcome measures $\mathrm{k}_{\mathrm{RFD}-\mathrm{SF}}, \mathrm{k}_{\mathrm{RFR}-\mathrm{SF}}, \mathrm{TP}_{\mathrm{RFD}}$, and $\mathrm{TP} \mathrm{RFR}_{\mathrm{R}}$, followed by Bonferroni-adjusted significance tests for post hoc pairwise comparisons. The inter-limb symmetry outcome measures were not normally distributed; therefore, a robust two-way ANOVA on trimmed means using the t2way function from the WRS2 package (Mair and Wilcox, 2020) was performed for betweengroup comparisons. When the main effect was found to be significant, the Yuen's modified test for independent trimmed means (20\% level) (Yuen, 1974) with a bootstrap $(n=599)$ was used for post hoc group comparisons (yuenbt function from the WRS2 package). The effect size for the two-way ANOVA of the main outcome measures was described using partial eta squared $\left(\eta_{p}^{2}\right)$ with the following interpretation: small (0.01-0.06), medium (0.06-0.14), and large (>0.14) (Cohen, 1992). Classical Cohen's $d$ effect size was calculated for post hoc comparisons (Cohen, 1988) of main outcome measures. For determining the magnitude of inter-limb symmetries by post hoc comparisons, a robust alternative to the classical Cohen's $d$ effect size with percentile bootstrap 95\% CI was calculated $\left(\delta_{R}\right)$ according to Algina et al. (2005). Both Cohen's $d$ and $\delta_{R}$ were interpreted as follows: negligible $(<0.2)$, small $(0.2-0.5)$, moderate $(0.5-0.8)$, and large $(>0.8)$. For all statistical tests performed, significance level was set at $p<0.05$ (two-tailed). 


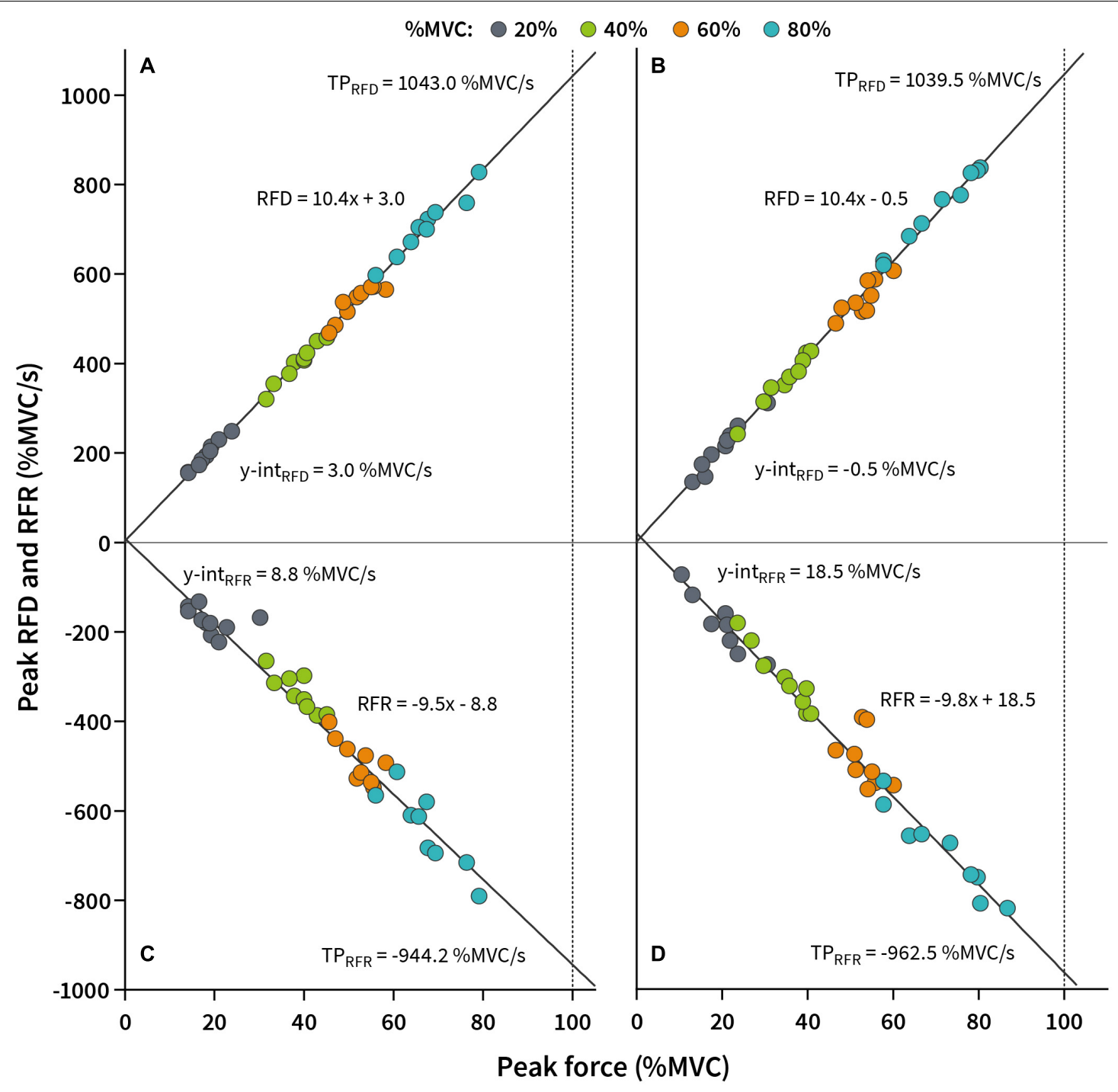

FIGURE 2 | The slope of the rate of force development/relaxation scaling factor ( $\mathrm{K}_{\mathrm{RFD}-\mathrm{SF}} / \mathrm{k}_{\mathrm{RFR}-\mathrm{SF}}$ ) of a representative participant interpolated to a data scatter. (A) Scatter plot and regression line of the nine randomly selected pulses at each submaximal level used for calculation of the $k_{R F D-S F}$ (B) Scatter plot and

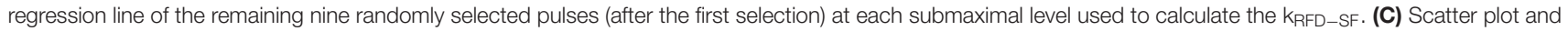
regression line of the nine randomly selected pulses at each submaximal level used to calculate the $k_{R F R-S F}$. (D) Scatter plot and regression line of the remaining

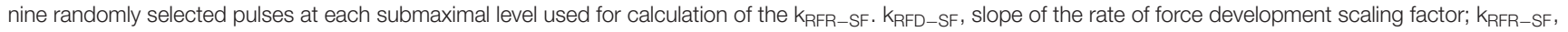

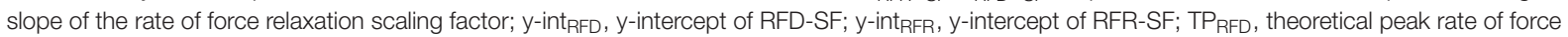
development; $\mathrm{TP}_{\mathrm{RFR}}$, theoretical peak rate of force relaxation.

\section{RESULTS}

\section{Reliability}

The first set of analysis examined the reliability of absolute outcome measures and outcome measures for inter-limb symmetry. Figure $\mathbf{2}$ displays the raw pulse data obtained during the RFD-SF/RFR-SF protocol for a single participant in the subsample study and demonstrates differences in outcome measures between randomly selected repetition clusters.

All the subsample reliability statistics are presented in Table 2. Overall, we found good to excellent relative $\left(\mathrm{ICC}_{2,1}\right)$ and excellent absolute reliability (CV) for the selected absolute main outcome measures $\left(\mathrm{k}_{\mathrm{RFD}-\mathrm{SF}}, \mathrm{k}_{\mathrm{RFR}-\mathrm{SF}}, \mathrm{TP}_{\mathrm{RFD}}\right.$, and $\left.\mathrm{TP}_{\mathrm{RFR}}\right)$ in both the preferred and non-preferred leg. Moderate to excellent relative and excellent absolute reliability was observed for symmetry main outcome measures.

\section{Gender and Sports Differences for Absolute Main Outcome Measures}

The effect of gender and sports on each of the absolute main outcome measures was examined by conducting a two-way ANOVA test. For both the preferred and non-preferred leg, there was no significant interaction between gender and sports on any of the main outcome measures (Table 3 ). 
TABLE 2 | Reliability results for absolute and inter-limb symmetry main outcome measures.

\begin{tabular}{|c|c|c|c|c|c|c|}
\hline & Outcome measure & Selection 1 & Selection 2 & ICC (95\% CI) & CV & TE \\
\hline \multirow[t]{8}{*}{ Preferred leg } & $\mathrm{k}_{\mathrm{RFD}-\mathrm{SF}}(/ \mathrm{s})$ & $9.1 \pm 1.1$ & $9.1 \pm 1.1$ & $0.96(0.92,0.98)$ & $2.5 \%$ & 0.20 \\
\hline & $\mathrm{k}_{\mathrm{RFR}-\mathrm{SF}}(/ \mathrm{s})$ & $-7.9 \pm 1.3$ & $-7.9 \pm 1.4$ & $0.94(0.89,0.97)$ & $4.2 \%$ & 0.30 \\
\hline & $r^{2}$ RFD-SF & $0.97 \pm 0.02$ & $0.97 \pm 0.02$ & $0.67(0.46,0.81)$ & $1.3 \%$ & 0.01 \\
\hline & $r^{2}$ RFR-SF & $0.90 \pm 0.09$ & $0.89 \pm 0.07$ & $0.82(0.69,0.90)$ & $3.6 \%$ & 0.03 \\
\hline & $y$-int $t_{R F D}(\% M V C / s)$ & $27.2 \pm 37.3$ & $25.8 \pm 33.9$ & $0.94(0.90,0.97)$ & * & 8.50 \\
\hline & $y$-int $t_{\text {RFR }}(\% M V C / s)$ & $-28.4 \pm 43.1$ & $-28.4 \pm 45.0$ & $0.91(0.84,0.95)$ & * & 13.60 \\
\hline & $\mathrm{TP}_{\mathrm{RFD}}(\% \mathrm{MVC} / \mathrm{s})$ & $932.8 \pm 83.6$ & $934.7 \pm 82.9$ & $0.97(0.94,0.98)$ & $1.6 \%$ & 15.20 \\
\hline & $\mathrm{TP}_{\mathrm{RFR}}(\% \mathrm{MVC} / \mathrm{s})$ & $-815.7 \pm 115.2$ & $-816.6 \pm 117.9$ & $0.96(0.94,0.98)$ & $2.7 \%$ & 22.40 \\
\hline \multirow[t]{8}{*}{ Non-preferred leg } & $\mathrm{k}_{\mathrm{RFD}-\mathrm{SF}}(/ \mathrm{s})$ & $8.8 \pm 1.2$ & $8.9 \pm 1.1$ & $0.95(0.90,0.97)$ & $2.9 \%$ & 0.30 \\
\hline & $\mathrm{k}_{\mathrm{RFR}-\mathrm{SF}}(/ \mathrm{s})$ & $-7.8 \pm 1.5$ & $-7.9 \pm 1.4$ & $0.91(0.83,0.95)$ & $5.7 \%$ & 0.50 \\
\hline & $r^{2}$ RFD-SF & $0.97 \pm 0.02$ & $0.97 \pm 0.04$ & $0.82(0.68,0.90)$ & $1.5 \%$ & 0.01 \\
\hline & $r^{2}$ RFR-SF & $0.89 \pm 0.11$ & $0.90 \pm 0.09$ & $0.83(0.71,0.90)$ & $5.0 \%$ & 0.04 \\
\hline & $y$-int RFD $(\% \mathrm{MVC} / \mathrm{s})$ & $25.4 \pm 30.5$ & $23.3 \pm 28.2$ & $0.90(0.82,0.95)$ & * & 9.30 \\
\hline & $y$-int & $-17.3 \pm 50.6$ & $-16.1 \pm 50.5$ & $0.87(0.77,0.93)$ & * & 18.70 \\
\hline & $\mathrm{TP}_{\mathrm{RFD}}(\% \mathrm{MVC} / \mathrm{s})$ & $905.3 \pm 91.8$ & $912.6 \pm 87.5$ & $0.96(0.93,0.98)$ & $1.9 \%$ & 17.50 \\
\hline & $\mathrm{TP}_{\mathrm{RFR}}(\% \mathrm{MVC} / \mathrm{s})$ & $-793.4 \pm 121.7$ & $-804.9 \pm 109.1$ & $0.94(0.89,0.97)$ & $3.5 \%$ & 27.90 \\
\hline \multirow[t]{4}{*}{ Symmetry (\%) } & $\mathrm{K}_{\mathrm{RFD}-\mathrm{SF}}(\%)$ & $91.6 \pm 5.1$ & $92.7 \pm 4.6$ & $0.78(0.63,0.88)$ & $2.4 \%$ & 2.20 \\
\hline & $\mathrm{k}_{\mathrm{RFR}-\mathrm{SF}}(\%)$ & $87.3 \pm 10.1$ & $87.6 \pm 10.3$ & $0.75(0.58,0.86)$ & $5.9 \%$ & 5.20 \\
\hline & TP $\mathrm{RFD}(\%)$ & $93.9 \pm 3.8$ & $94.6 \pm 3.6$ & $0.85(0.74,0.92)$ & $1.5 \%$ & 1.40 \\
\hline & $\mathrm{TP}_{\mathrm{RFR}}(\%)$ & $90.0 \pm 8.0$ & $90.3 \pm 8.0$ & $0.79(0.65,0.88)$ & $4.1 \%$ & 3.70 \\
\hline
\end{tabular}

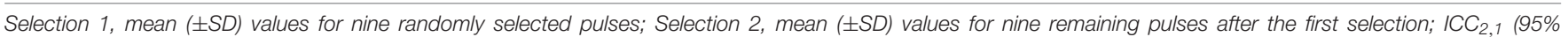

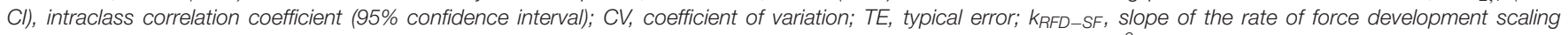

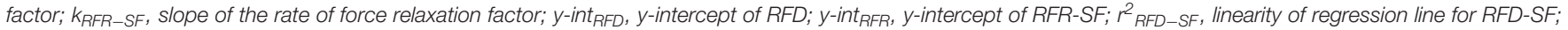

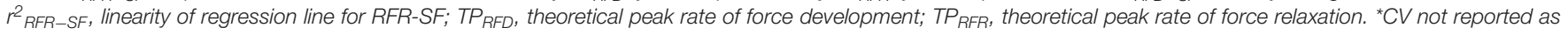
the $y$-intercept outcome measures contain both positive and negative values.

For the preferred leg, there was a significant main effect of gender on $\mathrm{k}_{\mathrm{RFD}-\mathrm{SF}}\left[F_{(1,242)}=17.03, p<0.001, \eta_{p}^{2}=0.06\right)$ and $\mathrm{TP}_{\mathrm{RFD}}\left[F_{(1,242)}=22.69, p<0.001, \eta_{p}^{2}=0.09\right]$ with higher $\mathrm{k}_{\mathrm{RFD}-\mathrm{SF}}(9.1 \pm 0.9$ vs. $8.6 \pm 0.9 / \mathrm{s}, d=0.57)$ and $\mathrm{TP}_{\mathrm{RFD}}$ (939.2 \pm 68.2 vs. $895.0 \pm 67.2 \% \mathrm{MVC} / \mathrm{s}, d=0.65)$ found in males compared to females (Figure 3). No main effect of sports on other main outcome measures was found for the preferred leg (Table 3).

For the non-preferred leg, a significant main effect of gender was found for $\mathrm{k}_{\mathrm{RFD}-\mathrm{SF}}\left[F_{(1,242)}=29.33, p<0.001, \eta_{p}^{2}=\right.$ $0.11]$ and $\mathrm{TP}_{\mathrm{RFD}}\left[F_{(1,242)}=32.29, p<0.001, \eta_{p}^{2}=0.12\right]$ (Table 3). Males showed significantly higher $\mathrm{k}_{\mathrm{RFD}-\mathrm{SF}}(9.1 \pm 0.9$ vs. $8.5 \pm 0.8 / \mathrm{s}, d=0.71)$ and $\mathrm{TP}_{\mathrm{RFD}}(931.0 \pm 67.0$ vs. $882.4 \pm 64.7 \% \mathrm{MVC} / \mathrm{s}, d=0.73$ ) compared to females (Figure 3), while no significant main effect of sports was observed on other main outcome measures.

\section{Gender and Sports Differences for Inter-Limb Symmetry Measures}

The effect of gender and sports on each of the main outcome measures was examined by conducting a robust nonparametric two-way ANOVA test. No significant interaction was found between gender and sports for any of the inter-limb symmetry outcome measures (Table 4). A significant main effect of gender was found for inter-limb symmetries in $\mathrm{k}_{\mathrm{RFR}-\mathrm{SF}}(p<0.05)$ and $\mathrm{TP}_{\mathrm{RFR}}(p<0.05)$. Yuen post hoc test revealed lower symmetry values in males for $\mathrm{k}_{\mathrm{RFR}-\mathrm{SF}}[88.4 \pm 8.6$ vs. $90.4 \pm 8.0 \%$, $p \leq 0.05, \delta_{\mathrm{R}}=0.31$ (95\% CI 0.06-0.54)] and TP $\mathrm{RFR}[90.9 \pm 6.8 \%$ vs. $92.5 \pm 6.0 \%, p \leq 0.05, \quad \delta_{\mathrm{R}}=0.29(95 \%$ CI $0.04-$ 0.52)] (Figure 4).

A significant main effect of sports was also found for $\mathrm{k}_{\mathrm{RFR}-\mathrm{SF}}(p<0.05)$ and $\mathrm{TP}_{\mathrm{RFR}}(p<0.01)$. Pairwise post hoc tests between individual sports groups showed that tennis players had significantly higher inter-limb symmetry in $\mathrm{k}_{\mathrm{RFR}-\mathrm{SF}}$ $(91.1 \pm 7.7 \%)$ compared to basketball players [88.4 \pm 8.7 , $p<0.05, \delta_{\mathrm{R}}=0.37$ (95\% CI 0.12-0.72)] and students [87.6 \pm 8.7 , $p<0.05, \delta_{\mathrm{R}}=0.52$ (95\% CI 0.20-0.97)] while the difference in $\mathrm{k}_{\mathrm{RFR}-\mathrm{SF}}$ symmetries between basketball players and students was not significant ( $p=0.46$ ) (Figure 5). Significantly higher values for inter-limb symmetry were also found in tennis players for $\mathrm{TP}_{\mathrm{RFR}}(93.1 \pm 6.0 \%)$ compared to basketball players $[90.9 \pm 6.7$, $p<0.01, \delta_{\mathrm{R}}=0.41$ (95\% CI 0.12-0.70)] and students [90.5 \pm 6.7 , $p<0.05, \delta_{\mathrm{R}}=0.50$ (95\% CI 0.12-0.93)] while no significant differences were found between basketball players and students $(p=0.69)$ (Figure 5).

\section{DISCUSSION}

The aim of the present study was to assess the reliability of the reduced RFD-SF/RFR-SF protocol for absolute outcome measures and the inter-limb symmetry measures calculated from the main absolute outcome measures ( $\mathrm{k}_{\mathrm{RFD}-\mathrm{SF}}, \mathrm{k}_{\mathrm{RFR}-\mathrm{SF}}$, $\mathrm{TP}_{\mathrm{RFD}}$, and $\left.\mathrm{TP}_{\mathrm{RFR}}\right)$. Additionally, we investigated differences for the main absolute and symmetry outcome measures across gender and sports. The analysis yielded the following results: 


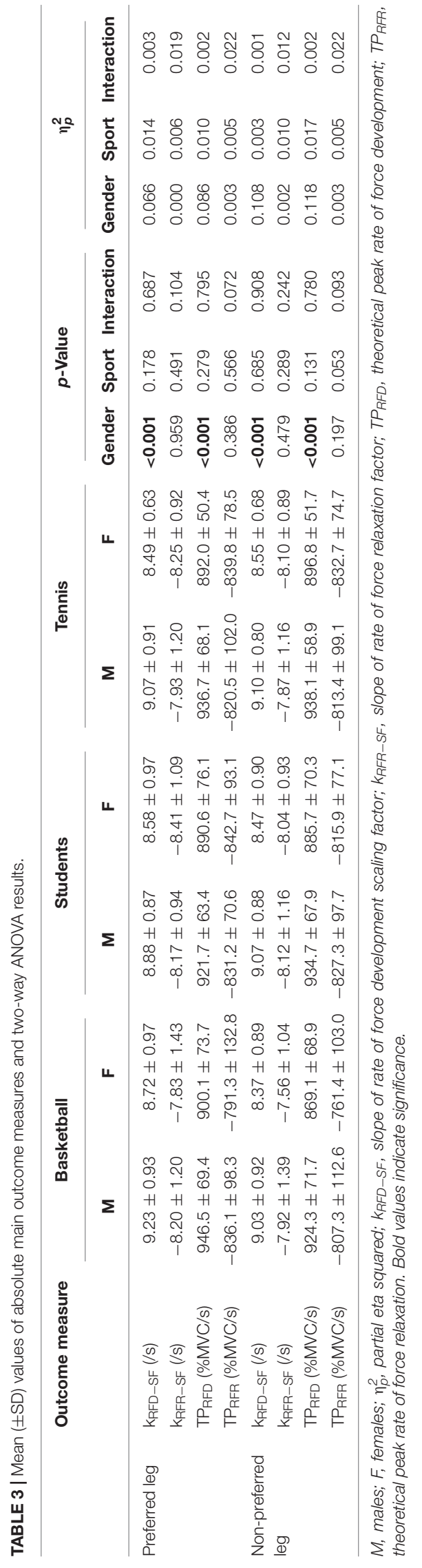

(1) good to excellent reliability for the main absolute outcome measures and moderate to excellent reliability for inter-limb symmetry outcome measures; (2) significantly higher absolute values for $\mathrm{k}_{\mathrm{RFD}-\mathrm{SF}}$ and $\mathrm{TP}_{\mathrm{RFD}}$ in males (both legs) compared to females, while there was no effect of sport; and (3) significantly lower symmetry values for $\mathrm{k}_{\mathrm{RFR}-\mathrm{SF}}$ and $\mathrm{TP}_{\mathrm{RFR}}$ in males compared to females and significantly higher symmetry values for $\mathrm{k}_{\mathrm{RFR}-\mathrm{SF}}$ and $\mathrm{TP}_{\mathrm{RFR}}$ for tennis players compared to basketball players and students.

Using two different subsets of pulses (36 pulses), excellent intra-session reliability was calculated for the main absolute outcome measures $\mathrm{k}_{\mathrm{RFD}-\mathrm{SF}}, \mathrm{TP}_{\mathrm{RFD}}$, and $\mathrm{TP}_{\mathrm{RFR}}$, while good to excellent reliability was found for $\mathrm{k}_{\mathrm{RFR}-\mathrm{SF}}$. As evidenced by a large TE found for outcome measures $y$-int $t_{\mathrm{RF}}$ and $y$-int $t_{R F R}$, the results of this study confirm observations of other authors about the y-intercept negligibility (Casartelli et al., 2014; Bellumori et al., 2017; Djordjevic and Uygur, 2017; Smajla et al., 2021b). Moderate to good reliability (moderate to excellent for $\mathrm{TP}_{\mathrm{RFD}}$ ) was observed for the RFD-SF/RFR-SF protocolbased inter-limb symmetry main outcome measures, enabling further comparisons between genders and different sports. The importance of the reliability of the inter-limb symmetry calculation method is highlighted by several previous studies, which observed significant variation in inter-limb symmetry and asymmetry values (Bishop et al., 2018a, 2019b) as poor reliability limits the practical application of study results. Based on the observed results, we can partially confirm our first hypothesis because most of the outcome measures including $\mathrm{y}$-intercepts showed good to excellent $\mathrm{ICC}_{2,1}$ values. Not all outcome measures showed satisfactory reliability as poor to good reliability with notably wider 95\% CI limits was found for $\mathrm{r}^{2}$ RFD-SF in the preferred leg and moderate to excellent reliability was found for $\mathrm{r}^{2} \mathrm{RFR}-\mathrm{SF}$ (preferred leg), $\mathrm{r}^{2}{ }_{\mathrm{RFD}}$-SF (non-preferred leg), and $\mathrm{r}^{2}$ RFR-SF (non-preferred leg). Despite the satisfactory relative agreement $\left(\mathrm{ICC}_{2,1}\right)$ for the y-intercept, high TEs would suggest that the use of this outcome measure is questionable.

Previous studies have suggested that RFD-SF/RFR-SF measures are independent of gender, and muscle size and strength (Bellumori et al., 2011; Djordjevic and Uygur, 2017). On this basis, we hypothesized that there would be no difference in the main absolute outcome measures between males and females. However, the analysis showed a significant effect of gender, as males showed significant greater values for $\mathrm{k}_{\mathrm{RFD}-\mathrm{SF}}$ $(9.1 \pm 0.9$ vs. $8.6 \pm 0.9 / \mathrm{s})$ and $\mathrm{TP}_{\mathrm{RFD}}(939.2 \pm 68.2$ vs. $895.0 \pm 67.2 \% \mathrm{MVC} / \mathrm{s})$ in the preferred and-non preferred leg ( $\mathrm{k}_{\mathrm{RFD}-\mathrm{SF}}: 9.1 \pm 0.9$ vs. $8.5 \pm 0.8 / \mathrm{s}$; $\mathrm{TP}_{\mathrm{RFD}}$ : $931.0 \pm 67.0$ vs. $882.4 \pm 64.7 \% \mathrm{MVC} / \mathrm{s})$. Prior studies using RFD-SF measures have confirmed a reduction in neuromuscular quickness with aging (Bellumori et al., 2013) and other impairments such as multiple sclerosis (Uygur et al., 2020) and knee osteoarthritis (Šarabon et al., 2020a), while there has been no evidence of the influence of gender on RFD-SF/RFR-SF outcome measures. Males and females in our study did not differ in age or training history which could have an influence on the observed results. When performing RFD-SF/RFR-SF calculations, the RFD is normalized to the participant's MVC, making this measure independent of muscle size and strength. For this reason, other 

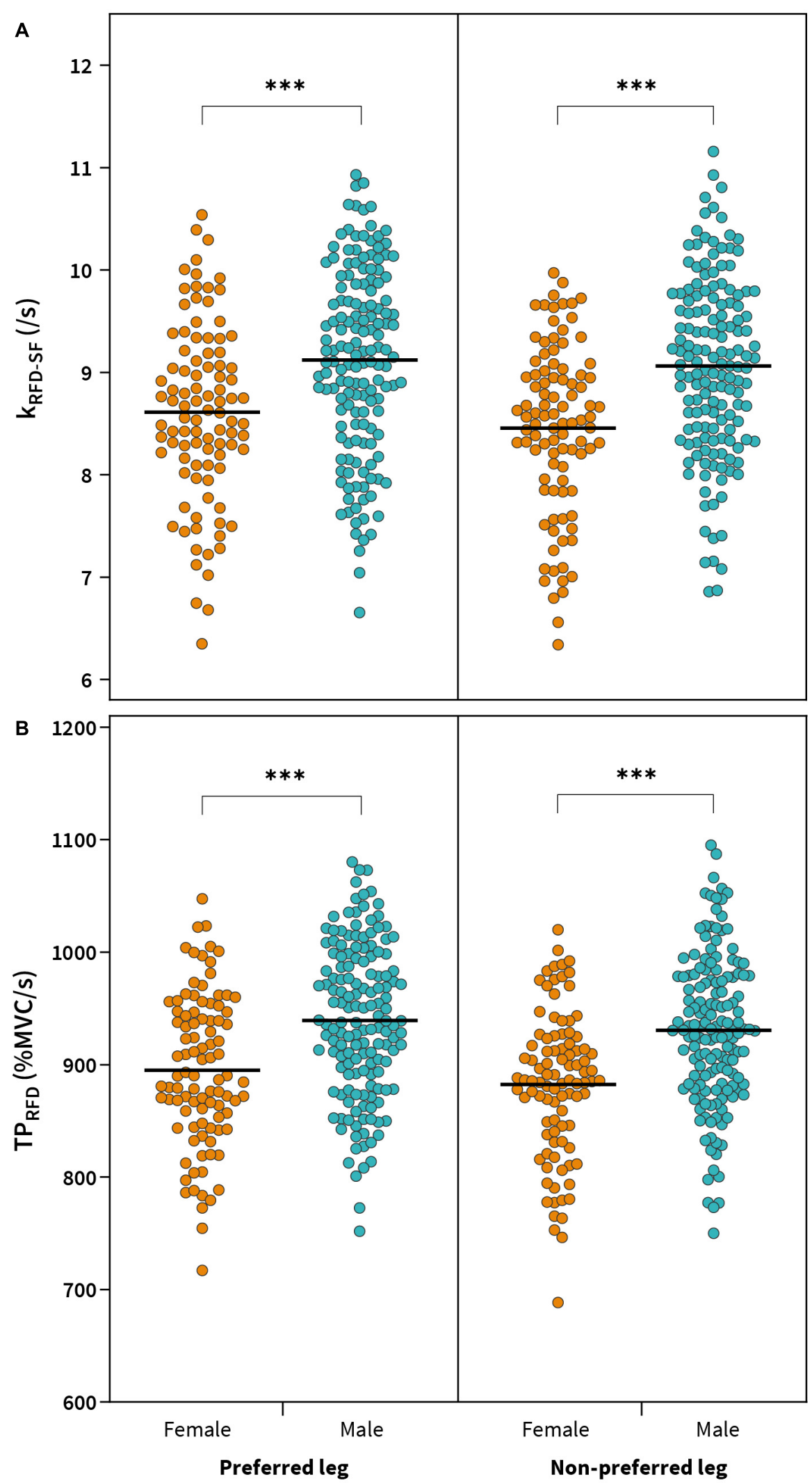

FIGURE 3 | Comparison between females and males for RFD-SF slope ( $\mathrm{k}_{\mathrm{RTD}-\mathrm{SF}}$ (A) and theoretical peak rate of force development (TP RFD (B). ${ }^{* * *} p<0.001$. 


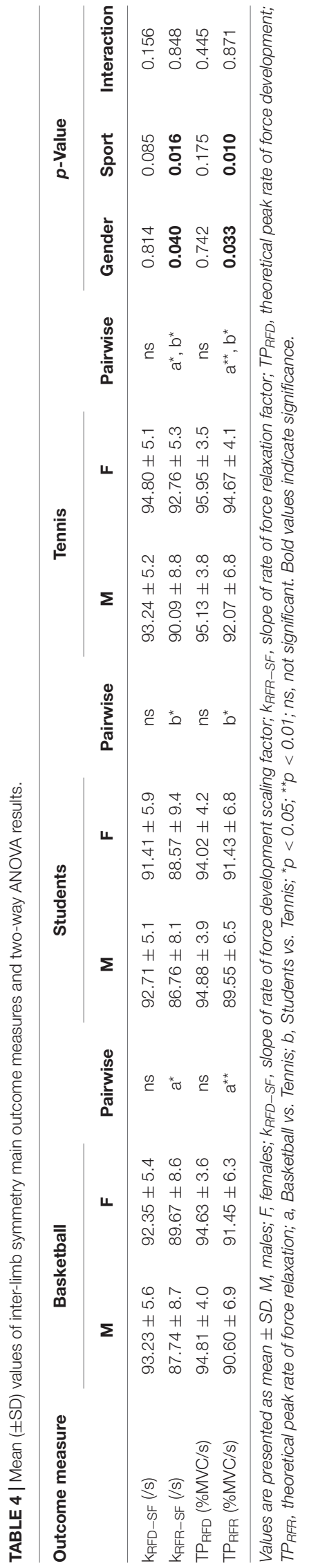

mechanisms might be responsible for the observed gender differences. In the early phase of force generation, a series of elastic components in the muscle-tendon complex must be stretched, which is related to the electromechanical delay (time from the onset of muscle activation until the onset of joint torque production) (Komi, 1984). Some previous studies have shown that males have a shorter electro-mechanical delay of the knee extensors compared to females (Bell and Jacobs, 1986; Zhou et al., 1995), while the stiffness and hysteresis of the tendon structure are lower in females compared to males (Kubo et al., 2003). This greater extensibility of tendon structures may cause a smaller transmission of contractile force on the joint and consequently on the maximal RFD at different submaximal intensities. This may partially explain greater $\mathrm{k}_{\mathrm{RFD}-\mathrm{SF}}$ and $\mathrm{TP}_{\mathrm{RFD}}$ in males compared to females. The differences between males and females occurred only for outcome measures that are related to force production $\left(k_{\mathrm{RFD}-\mathrm{SF}}\right.$ and $\left.\mathrm{TP}_{\mathrm{RFD}}\right)$. These measures are mainly dependent on neuromuscular activation mechanisms (Folland et al., 2014) such as double discharges and high firing rates (Klass et al., 2008), in addition to the mechanical properties of the muscle (contractile and connective tissue). Females have been shown to require greater neural drive to achieve fused tetanus during submaximal contractions compared to males (Inglis and Gabriel, 2020), which could further explain the gender differences observed in our study. On the other hand, the force relaxation mainly depends on the intrinsic properties of the muscle (Mathern et al., 2019). Even though it has been previously shown that females have a lower maximal rate of muscle relaxation (Amsterdam et al., 2008), these differences did not affect the RFR-SF outcome measures in our case.

Although we expected to find differences in our main outcome measures, there was no significant effect of sport. Basketball players showed the highest mean values of main outcome measures $\mathrm{k}_{\mathrm{RFD}-\mathrm{SF}}, \mathrm{k}_{\mathrm{RFR}-\mathrm{SF}}, \mathrm{TP}_{\mathrm{RFD}}$, and $\mathrm{TP}_{\mathrm{RFR}}$, while the smallest values were observed in students. However, these differences were not found to be significant. These results and the differences between genders allow us to reject our second hypothesis. This is the first study to evaluate the differences in RFD-SF/RFR-SF outcome measures between different sports. The results indicate that the type of training regimes that are typical of each of our studied groups does not affect the RFDSF/RFR-SF outcome measures. The differences were expected as one of the previous studies showed that knee extensor strength was greater in tennis players compared to basketball players (Šarabon et al., 2020b). However, this only refers to maximal capacity, whereas in the RFD-SF/RFR-SF protocol force production is evaluated at submaximal force production levels. Another possible explanation could be due to the young age of our sample in which distinct adaptations induced by specific training regimes are not yet apparent.

Inter-limb (a)symmetry quantification has been widely used in sports as it has been associated with sports performance (Bishop et al., 2019a; Madruga-Parera et al., 2019) and injury risk (Murphy et al., 2003; Lehance et al., 2009). Despite a large number of studies on inter-limb (a)symmetries, little is known to date about their variation between different sports. Some prior studies 

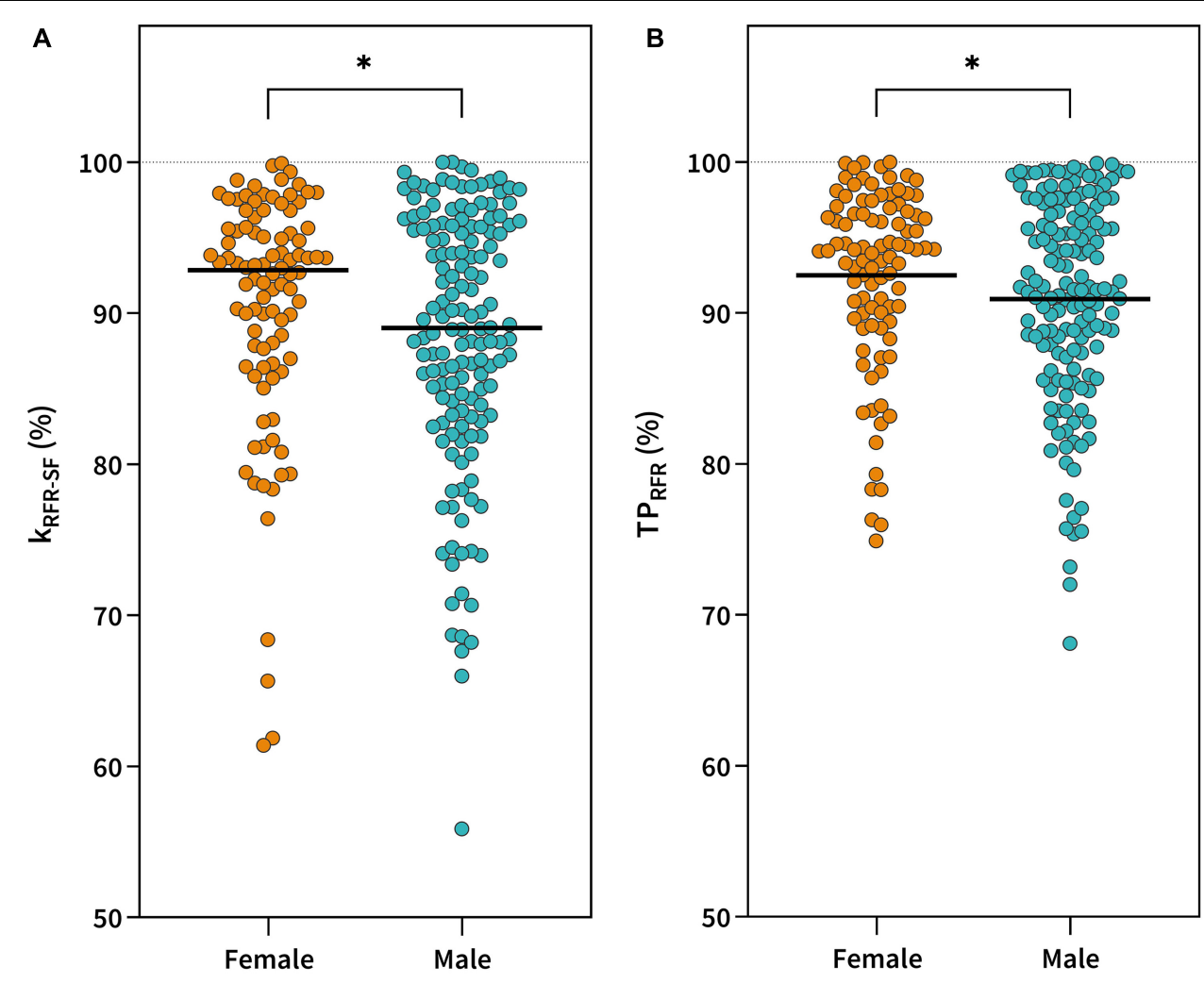

FIGURE 4 | Gender comparison in symmetry values for RFR-SF slope ( $\mathrm{k}_{\mathrm{RFR}-\mathrm{SF}}$ ) (A) and theoretical peak rate of force relaxation $(\mathrm{TP} \mathrm{RFR})$ (B). ${ }^{*} p<0.05$.

have mainly assessed maximal capacities (e.g., maximal strength, power, and speed) (Dos'Santos et al., 2018; Bishop et al., 2019a; Šarabon et al., 2020b). With the RFD-SF/RFR-SF protocol, we aim to assess rapid force production and relaxation across different ranges of submaximal intensities that are highly relevant to sports performance. To date, there are only two studies that have investigated inter-limb (a)symmetries with RFD-SF protocol, while the evaluation of (a)symmetries during the relaxation phase has not yet been reported. Boccia et al. (2018) showed that the prevalence of asymmetry was greater for $\mathrm{k}_{\mathrm{RFD}-\mathrm{SF}}$ compared to the classical isokinetic test in young soccer players. They found lower symmetry values for knee extensors $(82.6 \pm 9.1 \%)$ compared to male basketball players (93.23 $\pm 5.6 \%$ ), male tennis players $(92.71 \pm 5.1 \%)$, and male students $(93.24 \pm 5.2 \%)$ in our sample, which could be explained by a more one-sided nature of soccer (kicking with the preferred leg) compared to the sports included in our sample. In the second study, the authors calculated the $\mathrm{k}_{\mathrm{RFD}-\mathrm{SF}}$ of knee extensors in young athletes and reported that $15 \%$ of subjects could be classified as asymmetric (Smajla et al., 2020). In the current literature, there is no data about differences in (a)symmetries using the RFD-SF/RFR-SF protocol between different sports groups or genders. Moreover, we added additional outcome symmetry measures regarding $\mathrm{TP}_{\mathrm{RFD}}$ and $\mathrm{TP}_{\mathrm{RFR}}$. Our results revealed significantly lower values of symmetry in males compared to females for $\mathrm{k}_{\mathrm{RFR}-\mathrm{SF}}(88.4 \pm 8.6$ vs. $90.4 \pm 8.0 \%)$ and $\mathrm{TP}_{\mathrm{RFR}}$ (90.9 \pm 6.8 vs. $92.5 \pm 6.0 \%$ ) (Figure 4 ), while symmetry values for $\mathrm{k}_{\mathrm{RFD}-\mathrm{SF}}$ and $\mathrm{TP}_{\mathrm{RFR}}$ were similar (Table 4). Moreover, we found a significant effect of sport. Tennis players showed higher inter-limb symmetries in $\mathrm{k}_{\mathrm{RFR}-\mathrm{SF}}(91.1 \pm 7.7 \%)$ compared to basketball players $\left(88.4 \pm 8.7, p<0.05, \delta_{\mathrm{R}}=0.37\right)$ and students $(87.6 \pm 8.7 \%)$, while the same was found for $\mathrm{TP}_{\mathrm{RFR}}$ (tennis: $93.1 \pm 6.0 \%$, basketball: $90.9 \pm 6.7 \%$, and students: $90.5 \pm 6.7 \%)$. It is well established that daily tasks and sports activities require fine motor control of muscle contraction and relaxation (Kato et al., 2019). This is the first study that evaluated symmetry outcome measures using the RFD-SF/RFRSF protocol between genders and different sports groups. It seems that the outcome parameters of relaxation phase of the contraction are more sensitive to detect differences between our gender and sports groups. This can be supported with the study of Ohtaka and Fujiwara (2019), in which it was shown that the control error from the desired target force level was significantly greater for muscle relaxation than for contraction. Furthermore, it seems that muscle relaxation is more specific than contraction. For example, a deficit in muscle relaxation has been demonstrated in novice badminton players compared to skilled players and can be reduced with training (Sakurai and Ohtsuki, 2000). If one of the legs is used less frequently during specific sports movements, its relaxation ability may be reduced which can explain lower symmetry values. Tennis players perform a substantial amount of lateral movement on both sides (preferred and non-preferred), while basketball players use one leg more frequently because of specialized playing roles, 

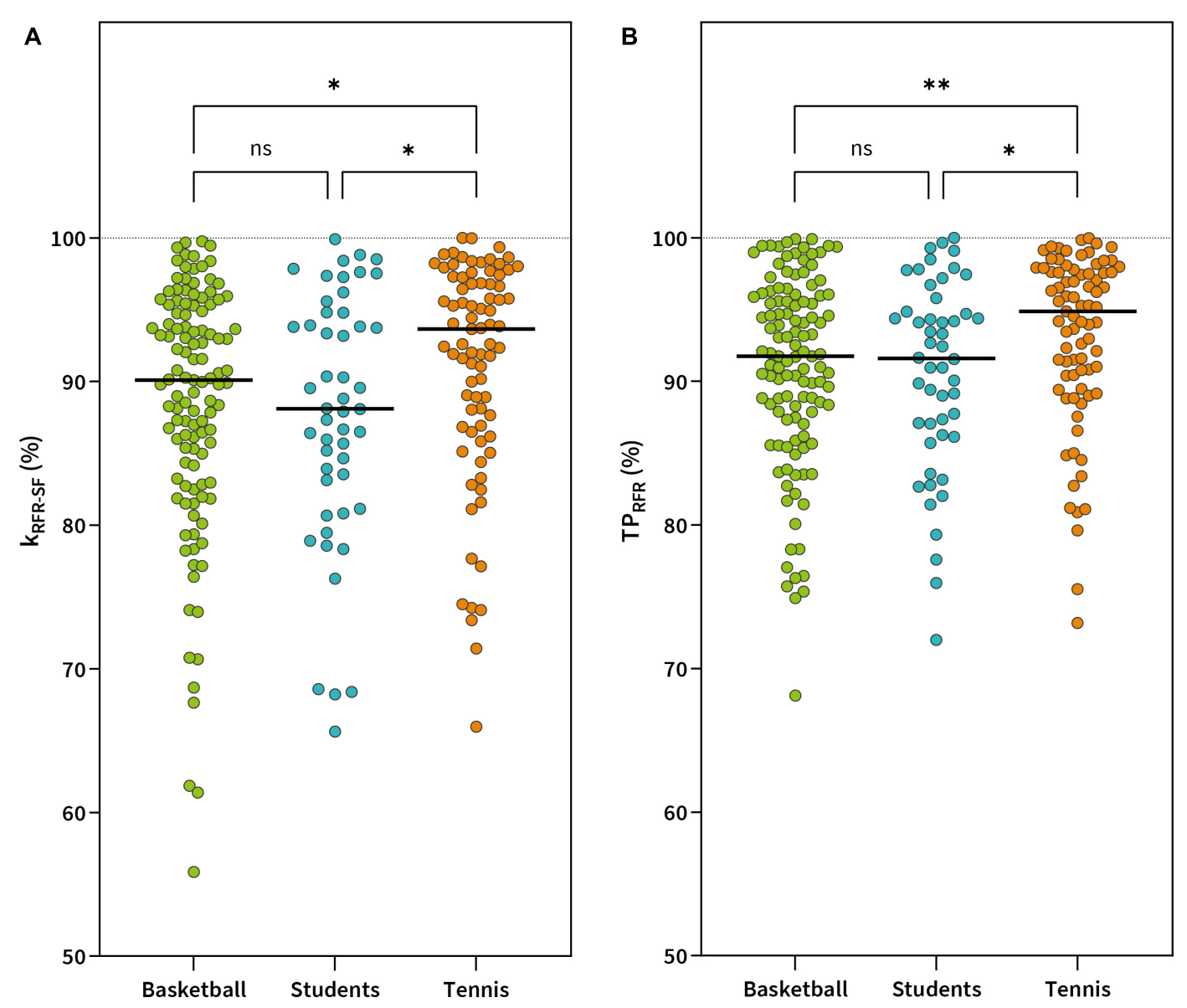

FIGURE 5 | Between sports comparison of symmetry values for RFR-SF slope ( $\mathrm{K}_{\mathrm{RFR}-\mathrm{SF}}$ (A) and theoretical peak rate of force relaxation (TPRFR) (B). ${ }^{* *} p<0.01$; $* p<0.05$; ns, not significant.

which could be the possible reason for lower symmetry values. Lower symmetry values in students compared to tennis players are difficult to explain as students perform a variety of sports activities which may have different influences on lower-limb symmetry abilities.

There are some limitations regarding the age and gender in our sample. Even though the strength of our study is a large number of participants, our sample size was not gender-balanced. Moreover, the students' group was slightly older compared to basketball and tennis players, while their sports activities vary between participants which may have a different influence on inter-limb symmetry. Nevertheless, this was the first study in the field that compared inter-limb symmetries using the RFDSF/RFR-SF protocol to examine gender and sports differences. To obtain more valuable information, future studies should compare a wider range of sports and compare the sensitivity of established outcome measures for (a)symmetry calculation relative to RFDSF/RFR-SF outcome measures.

Evaluation of submaximal force production and relaxation is important for all functional and sports movements. Our results suggest good to excellent intra-session reliability of the reduced RFD-SF/RFR-SF protocol which could be used for inter-limb symmetry quantification. The advantage of the RFD-SF/RFR-SF protocol is that force production and relaxation ability are tested across a wide span of submaximal intensities. Moreover, the relaxation phase of muscle contraction is often overlooked, although it was shown to be more sensitive for detecting various impairments. The sensitivity of RFD-SF/RFR-SF-derived measures should be further explored in future studies to confirm their use for inter-limb asymmetry investigation.

\section{DATA AVAILABILITY STATEMENT}

The raw data supporting the conclusions of this article will be made available by the authors, without undue reservation.

\section{ETHICS STATEMENT}

The studies involving human participants were reviewed and approved by the Slovenian Medical Ethics Committee (approval no. 0120-99/2018/5). Written informed consent to 
participate in this study was provided by the participants' legal guardian/next of kin.

\section{AUTHOR CONTRIBUTIONS}

NŠ conceptualized the idea and overviewed the measurement procedures and administration. DS and JŽ collected the data. JŽ analyzed the collected data. DS wrote the manuscript. NŠ and JŽ finalized the manuscript. All authors contributed to the article and approved the submitted version.

\section{REFERENCES}

Algina, J., Keselman, H., and Penfield, R. (2005). An alternative to Cohen's standardized mean difference effect size: a robust parameter and confidence interval in the two independent groups case. Psyhol. Methods 10, 317-328. doi: 10.1037/1082-989X.10.3.317

Amsterdam, V. U., Morse, C., Haan, A., De Amsterdam, V. U., and Jones, D. A. (2008). Sex differences in contractile properties and fatigue resistance of human skeletal muscle. Exp. Physiol. 93, 843-850. doi: 10.1113/expphysiol.2007. 041764

Bell, D., and Jacobs, I. (1986). Electro-mechanical response times and rate of force development in males and females. Med. Sci. Sport Exerc. 18, 31-36.

Bellumori, M., Jaric, S., and Knight, C. A. (2011). The rate of force development scaling factor (RFD-SF): protocol, reliability, and muscle comparisons. Exp. Brain Res. 212, 359-369. doi: 10.1007/s00221-011-2735-7

Bellumori, M., Jaric, S., and Knight, C. A. (2013). Age-related decline in the rate of force development scaling factor. Motor Control 17, 370-381. doi: 10.1123/mcj. 17.4.370

Bellumori, M., Uygur, M., and Knight, C. (2017). High-speed cycling intervention improves rate-dependent mobility in older adults. Med. Sci. Sport Exerc. 49, 106-114. doi: 10.1249/MSS.0000000000001069

Bishop, C., Abbot, W., Brashill, C., Turner, A., Lake, J., and Read, P. (2020). Bilateral vs. unilateral countermovement jumps: comparing the magnitude and direction of asymmetry in elite academy soccer players. J. Strength Cond. Res. doi: 10.1519/JSC.0000000000003679 [Epub Ahead of Print].

Bishop, C., Lake, J., Irineu, L., Papandopoulos, K., Turner, A., and Read, P. (2018a). Interlimb asymmetries: the need for an individual approach to data analysis. J. Strength Cond. Res. 35, 695-701. doi: 10.1519/JSC.0000000000002729

Bishop, C., Read, P., Brazier, J., Jarvis, P., Chavda, S., Bromley, T., et al. (2019a). Effects of interlimb asymmetries on acceleration and change of direction speed: a between-sport comparison of professional soccer and cricket athletes. J. Strength Cond. Res. doi: 10.1519/jsc.0000000000003135 [Epub ahead of print].

Bishop, C., Read, P., Chavda, S., Jarvis, P., and Turner, A. (2019b). Using unilateral strength, power and reactive strength tests to detect the magnitude and direction of asymmetry: a test-retest design. Sports 7:58. doi: 10.3390/ sports 7030058

Bishop, C., Turner, A., and Read, P. (2018b). Effects of inter-limb asymmetries on physical and sports performance: a systematic review. J. Sports Sci. 36, 1135-1144. doi: 10.1080/02640414.2017.1361894

Bishop, C., Turner, A., Maloney, S., Lake, J., Loturco, I., Bromley, T., et al. (2019c). Drop jump asymmetry is associated with reduced sprint and changeof-direction speed performance in adult female soccer players. Sports 7:29. doi: 10.3390/sports7010029

Boccia, G., Brustio, P. R., Buttacchio, G., Calabrese, M., Bruzzone, M., Casale, R., et al. (2018). Interlimb asymmetries identified using the rate of torque development in ballistic contraction targeting submaximal torques. Front. Physiol. 9:1-10. doi: 10.3389/fphys.2018.01701

Casartelli, N. C., Lepers, R., and Maffiuletti, N. A. (2014). Assessment of the rate of force development scaling factor for the hip muscles. Muscle Nerve 50, 932-938. doi: 10.1002/mus.24229

\section{FUNDING}

The study was supported by the Slovenian Research Agency through the project TELASI-PREVENT (L5-1845) (body asymmetries as a risk factor in musculoskeletal injury development: studying etiological mechanisms and designing corrective interventions for primary and tertiary preventive care) and research program fund P5-0147 Kinesiology of Monostructural, Polystructural and Conventional Sports. The funder played no role in conceptualization of the study, data acquisition, article writing, or any other phase of the study.

Cohen, J. (1988). Statistical Power Analysis for the Behavioral Sciences. New York, NY: Routlege Academic.

Cohen, J. (1992). A power primer. Psychol. Bull. 112, 155-159. doi: 10.1037//00332909.112.1.155

Cormack, S., Newton, R., McGuigan, M., and Doyle, T. (2008). Reliability of measures obtained during single and repeated countermovement jumps. Int. J. Sport Physiol. 3, 131-144. doi: 10.1123/ijspp.3.2.131

Croisier, J. L., Ganteaume, S., Binet, J., Genty, M., and Ferret, J. M. (2008). Strength imbalances and prevention of hamstring injury in professional soccer players: a prospective study. Am. J. Sports Med. 36, 1469-1475. doi: 10.1177/ 0363546508316764

Croisier, J. L., Malnati, M., Reichard, L. B., Peretz, C., and Dvir, Z. (2007). Quadriceps and hamstring isokinetic strength and electromyographic activity measured at different ranges of motion: a reproducibility study. J. Electromyogr. Kinesiol. 17, 484-492. doi: 10.1016/j.jelekin.2006.04.003

Djordjevic, D., and Uygur, M. (2017). Methodological considerations in the calculation of the rate of force development scaling factor. Physiol. Meas. 39:015001. doi: 10.1088/1361-6579/aa9f51

Dos'Santos, T., Christopher, T., Comfort, P., and Jones, P. A. (2018). Comparison of change of direction speed performance and asymmetries between team-sport athletes: application of change of direction deficit. Sports 6:174. doi: 10.3390/ sports6040174

Dos'Santos, T., Thomas, C. A., Jones, P., and Comfort, P. (2017). Asymmetries in single and triple hop are not detrimental to change of direction speed. J. Trainology 6, 35-41. doi: 10.17338/trainology.6.2_35

Dos'Santos, T., Thomas, C., Jones, P. A., and Comfort, P. (2019). Assessing asymmetries in change of direction speed performance: application of change of direction deficit. J. strength Cond. Res. 33, 2953-2961. doi: 10.1519/JSC. 0000000000002438

Folland, J. P., Buckthorpe, M. W., and Hannah, R. (2014). Human capacity for explosive force production: neural and contractile determinants. Scand. J. Med. Sci. Sport. 24, 894-906. doi: 10.1111/sms.12131

Fousekis, K., Tsepis, E., and Vagenas, G. (2010). Multivariate isokinetic strength asymmetries of the knee and ankle in professional soccer players. J. Sport. Med. Phys. Fit. 50, 465-474.

Gordon, J., and Ghez, C. (1987). Trajectory control in targeted force impulses. II. Pulse height control. Exp. Brain Res. 1, 241-252. doi: 10.1007/bf00248546

Grabiner, M. D. (1994). Maximum rate of force development is increased by antagonist conditioning contraction. J. Appl. Physiol. 77, 807-811. doi: 10.1152/ jappl.1994.77.2.807

Green, B., Bourne, M. N., and Pizzari, T. (2018). Isokinetic strength assessment offers limited predictive validity for detecting risk of future hamstring strain in sport: a systematic review and meta-analysis. Br. J. Sports Med. 52, 329-336. doi: 10.1136/bjsports-2017-098101

Hopkins, W. G. (2000). Measures of reliability in sports medicine and science. Sport. Med. 30, 1-15.

Inglis, J. G., and Gabriel, D. A. (2020). Sex differences in motor unit discharge rates at maximal and submaximal levels of force output. Appl. Physiol. Nutr. Metab. 45, 1197-1207. doi: 10.1139/apnm-2019-0958

Kamimura, T., Yoshioka, K., Ito, S., and Kusakabe, T. (2009). Increased rate of force development of elbow flexors by antagonist conditioning contraction. Hum. Mov. Sci. 28, 407-414. doi: 10.1016/j.humov.2009.02.005 
Kato, K., Vogt, T., and Kanosue, K. (2019). Brain activity underlying muscle relaxation. Front. Physiol. 10:1457. doi: 10.3389/fphys.2019.01457

Klass, M., Baudry, S., and Duchateau, J. (2008). Age-related decline in rate of torque development is accompanied by lower maximal motor unit discharge frequency during fast contractions. J. Appl. Physiol. 104, 739-746. doi: 10.1152/ japplphysiol.00550.2007

Knezevic, O. M., Mirkov, D. M., Kadija, M., Nedeljkovic, A., and Jaric, S. (2014). Asymmetries in explosive strength following anterior cruciate ligament reconstruction. Knee 21, 1039-1045. doi: 10.1016/j.knee.2014.07.021

Komi, P. (1984). Physiological and biomechanical correlates of muscle function: effects of muscle structure and stretch-shortening cycle on force and speed. Exerc. Sport Sci. Rev. 12, 81-121.

Koo, T. K., and Li, M. Y. (2015). A guideline of selecting and reporting intraclass correlation coefficients for reliability research. J. Chiropr. Med. 15, 155-163. doi: $10.1016 /$ j.jcm.2016.02.012

Kubo, K., Kanehisa, H., and Fukunaga, T. (2003). Gender differences in the viscoelastic properties of tendon structures. Eur. J. Appl. Physiol. 88, 520-526. doi: 10.1007/s00421-002-0744-8

Lehance, C., Binet, J., Bury, T., and Croisier, J. L. (2009). Muscular strength, functional performances and injury risk in professional and junior elite soccer players. Scand. J. Med. Sci. Sport. 19, 243-251. doi: 10.1111/j.1600-0838.2008. 00780.x

Lockie, R. G., Callaghan, S. J., Berry, S. P., Cooke, E. R. A., Jordan, C. A., Luczo, T. M., et al. (2014). Relationship between unilateral jumping ability and asymmetry on multidirectional speed in team-sport athletes. J. Strength Cond. Res. 28, 3557-3566. doi: 10.1519/JSC.0000000000000588

Madruga-Parera, M., Bishop, C., Fort-Vanmeerhaeghe, A., Beltran-Valls, M., Skok, O., and Romero-Rodríguez, D. (2019). Interlimb asymmetries in youth tennis players: relationships with performance. J. Strength Cond. Res. 34, 2815-2823. doi: 10.1519/JSC.0000000000003152

Maffiuletti, N. A., Bizzini, M., Widler, K., and Munzinger, U. (2010). Asymmetry in quadriceps rate of force development as a functional outcome measure in TKA. Clin. Orthop. Relat. Res. 468, 191-198. doi: 10.1007/s11999-009-0978-4

Maffiuletti, N., Bizzini, M., Desbrosses, K., Babault, N., and Munzinger, U. (2007). Reliability of knee extension and flexion measurements using the Con-Trex isokinetic dynamometer. Clin. Physiol. Funct. Imaging. 27, 346-353. doi: 10. 1111/j.1475-097X.2007.00758.x

Mair, P., and Wilcox, R. (2020). Robust statistical methods in R using the WRS2 package. Behav. Res. Methods 52, 464-488. doi: 10.3758/s13428-019-01246-w

Maloney, S. J., Fletcher, I. M., and Richards, J. (2016). A comparison of methods to determine bilateral asymmetries in vertical leg stiffness. J. Sports Sci. 34, 829-835. doi: 10.1080/02640414.2015.1075055

Mathern, R. M., Anhorn, M., and Uygur, M. (2019). A novel method to assess rate of force relaxation: reliability and comparisons with rate of force development across various muscles. Eur. J. Appl. Physiol. 119, 291-300. doi: 10.1007/s00421018-4024-7

Mirkov, D. M., Knezevic, O. M., Maffiuletti, N. A., Kadija, M., Nedeljkovic, A., and Jaric, S. (2017). Contralateral limb deficit after ACL-reconstruction: an analysis of early and late phase of rate of force development. J. Sports Sci. 35, 435-440. doi: 10.1080/02640414.2016.1168933

Murphy, D. F., Connolly, D. A. J., and Beynnon, B. D. (2003). Risk factors for lower extremity injury: a review of the literature. Br. J. Sports Med. 37, 13-29. doi: 10.1136/bjsm.37.1.13

Ohtaka, C., and Fujiwara, M. (2019). Force control characteristics for generation and relaxation in the lower limb. J. Mot. Behav. 51, 331-341. doi: 10.1080/ 00222895.2018.1474337
Sakurai, S., and Ohtsuki, T. (2000). Muscle activity and accuracy of performance of the smash stroke in badminton with reference to skill and practice. J. Sports Sci. 18, 901-914. doi: 10.1080/026404100750017832

Šarabon, N., Čeh, T., Kozinc, Ž, and Smajla, D. (2020a). Adapted protocol of rate of force development and relaxation scaling factor for neuromuscular assessment in patients with knee osteoarthritis. Knee 27, 1697-1707. doi: 10.1016/j.knee. 2020.09 .023

Sarabon, N., Rosker, J., Fruhmann, H., Burggraf, S., Loefler, S., and Kern, H. (2013). Reliability of maximal voluntary contraction related parameters measured by a novel portable isometric knee dynamometer. Phys. Medizin Rehabil. Kurortmedizin 23, 22-27. doi: 10.1055/s-0032-1331190

Šarabon, N., Smajla, D., Maffiuletti, N. A., and Bishop, C. (2020b). Strength, jumping and change of direction speed asymmetries in soccer, basketball and tennis players. Symmetry (Basel). 12, 1-13. doi: 10.3390/sym12101664

Sato, K., and Heise, G. (2012). Influence of weight distribution asymmetry on the biomechanics of a barbell back squat. J. Strenght. Cond. Res. 26, 342-349. doi: 10.1519/JSC.0b013e318220e0a3

Smajla, D., Knezevic, O. M., Mirkov, D. M., and Šarabon, N. (2020). Interlimb asymmetries and ipsilateral associations of plantar flexors and knee extensors rate-of-force development scaling factor. Symmetry (Basel) 12:1522. doi: 10. 3390/sym12091522

Smajla, D., Spudić, D., and Šarabon, N. (2021a). Influence of load and phase of contraction on lateral symmetries in flywheel squats. Symmetry (Basel). 13, 1-14. doi: $10.3390 /$ sym 13010111

Smajla, D., Žitnik, J., and Šarabon, N. (2021b). Advancements in the protocol for rate of force development/relaxation scaling factor evaluation. Front. Hum. Neurosci. 15:654443. doi: 10.3389/fnhum.2021.654443

Thorlund, J. B., Aagaard, P., and Madsen, K. (2009). Rapid muscle force capacity changes after soccer match play. Int. J. Sports Med. 30, 273-278. doi: 10.1055/s0028-1104587

Uygur, M., de Freitas, P. B., and Barone, D. A. (2020). Rate of force development and relaxation scaling factors are highly sensitive to detect upper extremity motor impairments in multiple sclerosis. J. Neurol. Sci. 408:116500. doi: 10. 1016/j.jns.2019.116500

Wilson, G. J., and Murphy, A. J. (1996). The use of isometric tests of muscular function in athletic assessment. Sport Med. 22, 19-37. doi: 10.2165/00007256199622010-00003

Yuen, K. (1974). The two-sample trimmed t for unequal population variances. Biometrika 61:165.

Zhou, S., Lawson, D., Morrison, W., and Fairweather, I. (1995). Electromechanical delay of knee extensors: the normal range and the effects of age and gender. J. Hum. Mov. Stud. 28, 127-146.

Conflict of Interest: NŠ was employed by the company S2P, Science to Practice d.o.o.

The remaining authors declare that the research was conducted in the absence of any commercial or financial relationships that could be construed as a potential conflict of interest.

Copyright (c) 2021 Smajla, Žitnik and Šarabon. This is an open-access article distributed under the terms of the Creative Commons Attribution License (CC BY). The use, distribution or reproduction in other forums is permitted, provided the original author(s) and the copyright owner(s) are credited and that the original publication in this journal is cited, in accordance with accepted academic practice. No use, distribution or reproduction is permitted which does not comply with these terms. 